\title{
Sodium valproate, a histone deacetylase inhibitor, augments the expression of cell-surface NKG2D ligands, MICA/B, without increasing their soluble forms to enhance susceptibility of human osteosarcoma cells to NK cell-mediated cytotoxicity
}

\author{
KOJI YAMANEGI ${ }^{*}$, JUNKO YAMANE ${ }^{1 *}$, KENTA KOBAYASHI $^{1}$, NAHOKO KATO-KOGOE $^{1}$, \\ HIDEKI OHYAMA $^{1}$, KEIJI NAKASHO ${ }^{1}$, NAOKO YAMADA ${ }^{1}$, MASAKI HATA ${ }^{1}$, TOSHIHIRO NISHIOKA ${ }^{1,2}$, \\ SATORU FUKUNAGA ${ }^{3}$, HIROYUKI FUTANI ${ }^{3}$, HARUKI OKAMURA ${ }^{4}$ and NOBUYUKI TERADA ${ }^{1}$ \\ Departments of ${ }^{1}$ Pathology, ${ }^{2}$ Dentistry and Oral Surgery, ${ }^{3}$ Orthopedic Surgery, and ${ }^{4}$ Laboratory of Host Defenses, \\ Institute for Advanced Medical Sciences, Hyogo College of Medicine, \\ 1-1 Mukogawa-cho, Nishinomiya Hyogo 663-8501, Japan
}

Received June 28, 2010; Accepted August 17, 2010

DOI: 10.3892/or_00001026

\begin{abstract}
MHC class I-related chain molecules A and B (MICA and B) expressed on the cell-surface of tumor cells are ligands for an activating receptor, $\mathrm{NKG} 2 \mathrm{D}$, expressed on natural killer (NK) cells and stimulate the NK cell-mediated cytotoxicity. On the other hand, the soluble form of MICA and $\mathrm{B}$ produced by proteolytic cleavage of cell-surface MIC interferes with NK cell-mediated cytotoxicity. We investigated effect of sodium valproate (VPA), a histone deacetylase inhibitor, on the production of cell-surface and soluble MICA and $\mathrm{B}$ and NK cell-mediated cytotoxicity in four human osteosarcoma cells. VPA at 0.5 and $1.0 \mathrm{mM}$ induced acetylation of histones bound to MICA and B gene promoters, increased cell-surface but not soluble MICA and B, and augmented the susceptibility of osteosarcoma cells to NK cell-mediated cytotoxicity. The present results indicate that VPA sensitizes human osteosarcoma cells to cytotoxicity of NK cells.
\end{abstract}

\section{Introduction}

Tumor cells express MHC class I-related chain molecules $A$ and B (MICA and B) on their cell-surface which are the ligands of an activating receptor, NKG2D, expressed on

Correspondence to: Dr Koji Yamanegi, Department of Pathology, Hyogo College of Medicine, 1-1 Mukogawa-cho, Nishinomiya, Hyogo 663-8501, Japan

E-mail: yamanegi@hyo-med.ac.jp

*Contributed equally

Key words: VPA, HDAC inhibitor, MIC, soluble MIC, osteosarcoma the cell-surface of natural killer (NK) cells. Binding of NK cells to tumor cells through interaction between NKG2D and MICA or B induces cytolysis of tumor cells $(1,2)$. Tumor cells also produce the soluble form of MICA and B by proteolytic cleavage of cell-surface MICA and B. Soluble MICA and B interfere with interaction between tumor and NK cells to prevent NK cell-mediated cytotoxicity (1-3). Therefore, in order to augment the susceptibility of tumor cells to NK cell cytotoxicity, it is necessary to increase the expression of cell-surface MICA and B or to decrease the production of the soluble form of MICA and B.

It has been shown that gene expression is regulated by acetylation and deacetylation of histones catalyzed by histone acetyltransferase and histone deacetylases (HDACs), respectively. HDAC inhibitors which alter the histone acetylation status have been shown to induce cell cycle arrest and apoptosis in various tumor cells $(4,5)$. In addition, HDAC inhibitors stimulate the expression of cellsurface MICA and B on tumor cells to enhance cytotoxicity of NK cells and host immune surveillance against tumor cells (6-11).

In the case of osteosarcoma, HDAC inhibitors enhance the susceptibility to Fas-ligand-induced cell death, indicating that host immune defense against osteosarcoma cells is also strengthened by HDAC inhibitors (12-14). However, whether the susceptibility of osteosarcoma cells to cytotoxicity of NK cells in the presence of HDAC inhibitors is due to altered production of cell-surface MICA and B or their soluble forms is not clear. In this study, we attempted to clarify this issue using sodium valproate (VPA) as an HDAC inhibitor. VPA was chosen since it has long been used for treatment of epilepsy and bipolar disorder without serious side effects $(15,16)$.

\section{Materials and methods}

Cells. MG-63, HOS, U-2 OS and SaOS-2 human osteosarcoma cells were cultured as described previously (13). 
Human natural killer NK-92 cells were purchased from American Type Culture Collection (ATCC, Manasas, VA, USA) and cultured in Minimum Essential alpha Medium (Invitrogen, Carlsbad, CA, USA) containing $0.2 \mathrm{mM}$ inositol, $0.1 \mathrm{mM}$ 2-mercaptoethanol, $0.02 \mathrm{mM}$ folic acid, $17 \mathrm{mM}$ sodium bicarbonate, $12.5 \%$ horse serum (Invitrogen), $12.5 \%$ fetal bovine serum (FBS) and $100 \mathrm{U} / \mathrm{ml}$ recombinant human interleukin 2 (Peprotech, Rock Hill, NJ, USA). All cells were cultured in a humidified atmosphere of $5 \% \mathrm{CO}_{2}$ in air at $37^{\circ} \mathrm{C}$.

Expression of MICA/B and HLA-A, B, C. MG-63, HOS, U-2 OS and SaOS- 2 cells were seeded at 2, 2, 2 and $4 \times 10^{4}$ cells/ dish, respectively, in $6-\mathrm{cm}$ tissue culture dishes containing $4 \mathrm{ml}$ of medium per dish. After $24 \mathrm{~h}$ (day 0), cells were cultured further in medium with $0,0.5$ or $1.0 \mathrm{mM}$ sodium valproate (VPA) (Wako, Osaka, Japan) with medium change on day 3. On day 3 or 7, cells were harvested and suspended in PBS containing $1 \%$ bovine serum albumin (BSA), and incubated with $100 \mathrm{ng} / \mathrm{ml}$ of PE-conjugated anti-human MICA/B mouse monoclonal antibody $\left(\operatorname{IgG}_{2 b}\right)$ (R\&D Systems, Minneapolis, MN, USA) or $100 \mathrm{ng} / \mathrm{ml}$ of control mouse $\mathrm{IgG}_{2 \mathrm{~b}}$ (BioLegend, San Diego, CA, USA), and $100 \mathrm{ng} / \mathrm{ml}$ of FITC-conjugated anti-human HLA-A, B, C mouse monoclonal antibody $\left(\operatorname{IgG}_{2 \mathrm{a}}\right.$ ) (BioLegend) or $100 \mathrm{ng} / \mathrm{ml}$ of control mouse $\operatorname{IgG}_{2 \mathrm{a}}$ (BioLegend) for $15 \mathrm{~min}$ on ice. After washing with $\mathrm{Ca}^{++}$and $\mathrm{Mg}^{++}$-free phosphate-buffered saline (PBS) containing $1 \% \mathrm{BSA}, 1 \times 10^{4}$ cells were analyzed using a BD FACSCalibur ${ }^{\mathrm{TM}}$ flow cytometer equipped with a CellQuest software (BD Biosciences, San Jose, CA, USA).

Production of soluble MICA and B. MG-63, HOS, U-2 OS and SaOS- 2 cells were seeded at $1,1,1$ and $2 \times 10^{5}$ cells/dish, respectively, in 10-cm tissue culture dishes containing $5 \mathrm{ml}$ of medium per dish. After $24 \mathrm{~h}$ (day 0), cells were cultured further in medium with $0,0.5$ and $1.0 \mathrm{mM}$ VPA. On day 3, half of cultures were analyzed for soluble MICA and B and viable cells. The other half of cultures exchanged fresh medium with or without VPA and were cultured further, and soluble MICA and B and viable cells were measured on day 7 . Viable cells were determined by the trypan blue exclusion test. For the assay of soluble MICA and B, after centifugation at $300 \mathrm{x}$ g for $5 \mathrm{~min}$, the medium was analyzed for MICA or B using a DuoSet ${ }^{\circledR}$ ELISA Development System for human MICA or B (R\&D Systems) and a microplate reader (Bio-Rad, Tokyo, Japan). The effect of VPA on the production of soluble MICA and B was evaluated by determining the amount of soluble MICA and B per $10^{4}$ viable cells in VPA-treated cultures as percentage of the average value in untreated cultures.

Cytotoxicity assay. Cytotoxicity of NK cells against HOS, U-2 OS and SaOS-2 osteosarcoma cells was analyzed using a CytoTox-Fluor ${ }^{\mathrm{TM}}$ Cytotoxicity Assay kit (Promega, Madison, WI, USA), according to the manufacturer's instructions. Osteosarcoma target cells (T) were cultured in medium with or without 0.5 or $1.0 \mathrm{mM}$ VPA for 7 days. Then, the NK cell cytotoxicity assay was carried out by incubating these cells with NK-92 effector cells (E) at various T:E ratios (1/20-1/5) for $3 \mathrm{~h}$ at $37^{\circ} \mathrm{C}$. In blocking experiments, NK-92 cells were cultured in medium with $1.0 \mu \mathrm{g} / \mathrm{ml}$ of purified anti-human NKG2D antibody (BioLegend) for $16 \mathrm{~h}$ before incubating with target cells. The number of dead cells was estimated by measuring the activity of proteases in medium which was released from dead cells. The protease activity in medium released spontaneously from target cells and the total maximum protease activity released from all target cells by treatment with $1 \%$ Triton $\mathrm{X}-100$ were also determined. The percentage of target cells killed by NK-92 cells was calculated as follows: $100 \mathrm{x}$ (sample protease activity - spontaneously released protease activity)/(total maximum protease activity spontaneously released protease activity).

Chromatin immunoprecipitation (ChIP) assay. The ChIP assay was carried out using an EZ-Magna ChIP ${ }^{\mathrm{TM}}$ kit (Millipore, Billerica, MA, USA) according to the manufacturer's instructions. U-2 OS and SaOS-2 cells were cultured in medium with or without $0.5 \mathrm{mM}$ or $1.0 \mathrm{mM}$ VPA for 7 days. After cross-linking of proteins bound to DNA with formaldehyde, nuclear lysate containing protein-DNA complexes was prepared from these cells. An aliquot of the nuclear lysate was used to immunoprecipitate acetylated histone-DNA complexes with anti-acetyl-histone $\mathrm{H} 3$ rabbit polyclonal antibody. DNA was extracted from the precipitated acetylated histone-DNA complexes and from another aliquot of nuclear lysate. MICA and MICB gene promoter sequences were amplified by polymerase chain reaction (PCR) using Takara Ex Taq ${ }^{\mathrm{TM}}$ DNA polymerase (Takara Bio Inc., Ohtsu, Shiga, Japan) and the following primers; sense, 5'-GCTTATGAAGTTGGAGCTGT-3', and antisense, 5'-AGCCAGAAGCAGGAAGAC-3', for MICA promoter, and sense, 5'-TCAGCTACTCGGGGCTGGTC-3', and antisense, 5'-ACGGCCAGAAACAGCAGGAC-3', for MICB promoter. The PCR conditions were $94^{\circ} \mathrm{C}$ for $1 \mathrm{~min}$, $53^{\circ} \mathrm{C}$ for $1 \mathrm{~min}$, and $72^{\circ} \mathrm{C}$ for $1 \mathrm{~min}$ for 35 cycles for MICA gene promoter, and $94^{\circ} \mathrm{C}$ for $1 \mathrm{~min}, 64^{\circ} \mathrm{C}$ for $1 \mathrm{~min}$, and $72^{\circ} \mathrm{C}$ for $1 \mathrm{~min}$ for 35 cycles for MICB gene promoter. The amplified fragments were resolved by electrophoresis in a $1.5 \%$ agarose gel and detected by ethidium bromide staining.

Statistical analysis. Data were presented as a mean \pm SE. Data of 3 groups or more were analyzed by the two-tailed Dunnett's t-test for multiple comparison. A P-value of $<0.05$ was considered significant.

\section{Results}

Effect of VPA on the expression of MICA/B and HLA class I on the surface of osteosarcoma cells. MG-63, HOS, U-2 OS and SaOS-2 cells were cultured in the presence or absence of VPA at 0.5 or $1.0 \mathrm{mM}$ for 3 or 7 days and the expression of cell surface MICA/B and HLA class I was examined by flow cytometry. All four osteosarcoma cell lines expressed MICA/B and HLA class I on their cell surface (Fig. 1). VPA at $0.5 \mathrm{mM}$ increased MICA/B-positive cells in U-2 OS and SaOS- 2 cells on day 3 and in all four osteosarcoma cells on day 7, while VPA at $1.0 \mathrm{mM}$ increased MICA/B-positive cells in all four osteosarcoma cells on days 3 and 7 (Fig. 2). The increases in MICA/B-positive cells in the presence of 
MICA and B
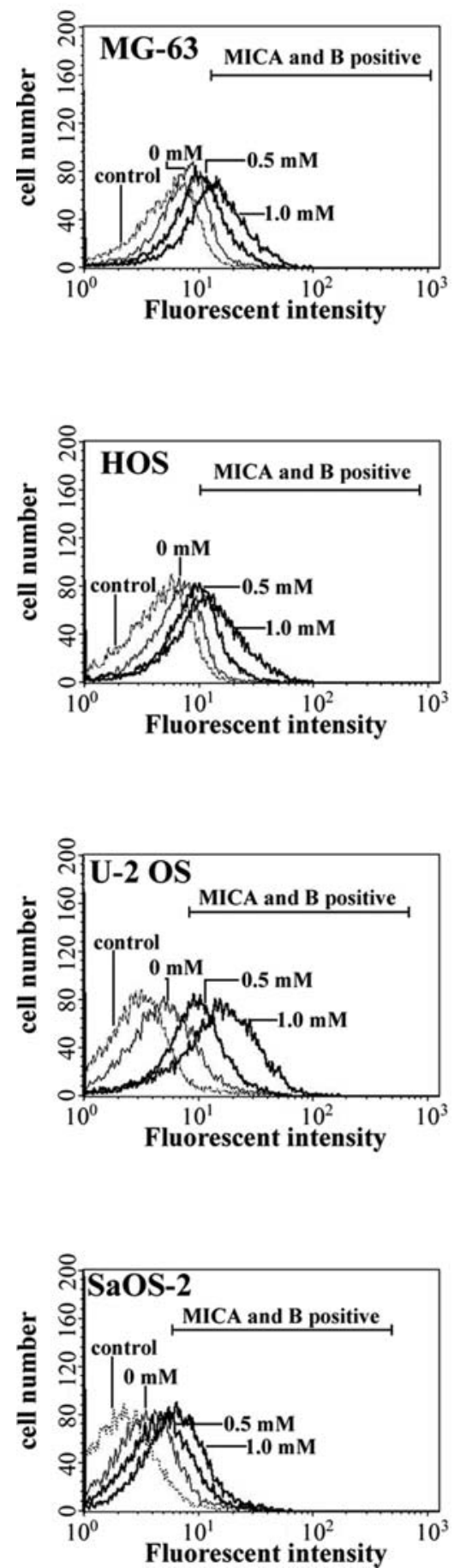

HLA


Figure 1. Flow cytometric analysis of the expression of cell-surface MICA and B and HLA class I. Representative profiles of four osteosarcoma cells cultured for 7 days in the presence or absence of VPA at 0.5 or $1.0 \mathrm{mM}$ are presented. The control indicates a profile of cells incubated with mouse IgG. Positive cells are indicated by the horizontal bar.

0.5 and 1.0 mM VPA on day 7 were 1.4-2.3- and 2.3-5.5-fold, respectively.

VPA at 0.5 and $1.0 \mathrm{mM}$ did not increase HLA class Ipositive cells in MG-63 cells on days 3 and 7, but significantly increased these cells in HOS cells on day 3, in U-2 OS cells on days 3 and 7, and in SaOS-2 cells on day 7 (Fig. 3).

Effect of VPA on acetylation of histones bound to MICA and MICB gene promoters. To determine whether upregulation of MICA and MICB expression by VPA is associated with acetylation of histones bound to the promoters of the MICA and MICB genes, the ChIP assay was carried out using U-2 OS and SaOS-2 cells. The results showed that VPA at 0.5 and $1.0 \mathrm{mM}$ enhanced acetylation of histones bound to the MICA and MICB promoters (Fig. 4).

Effect of VPA on the production of soluble MICA and B. To determine effect of VPA on the production of soluble MICA 

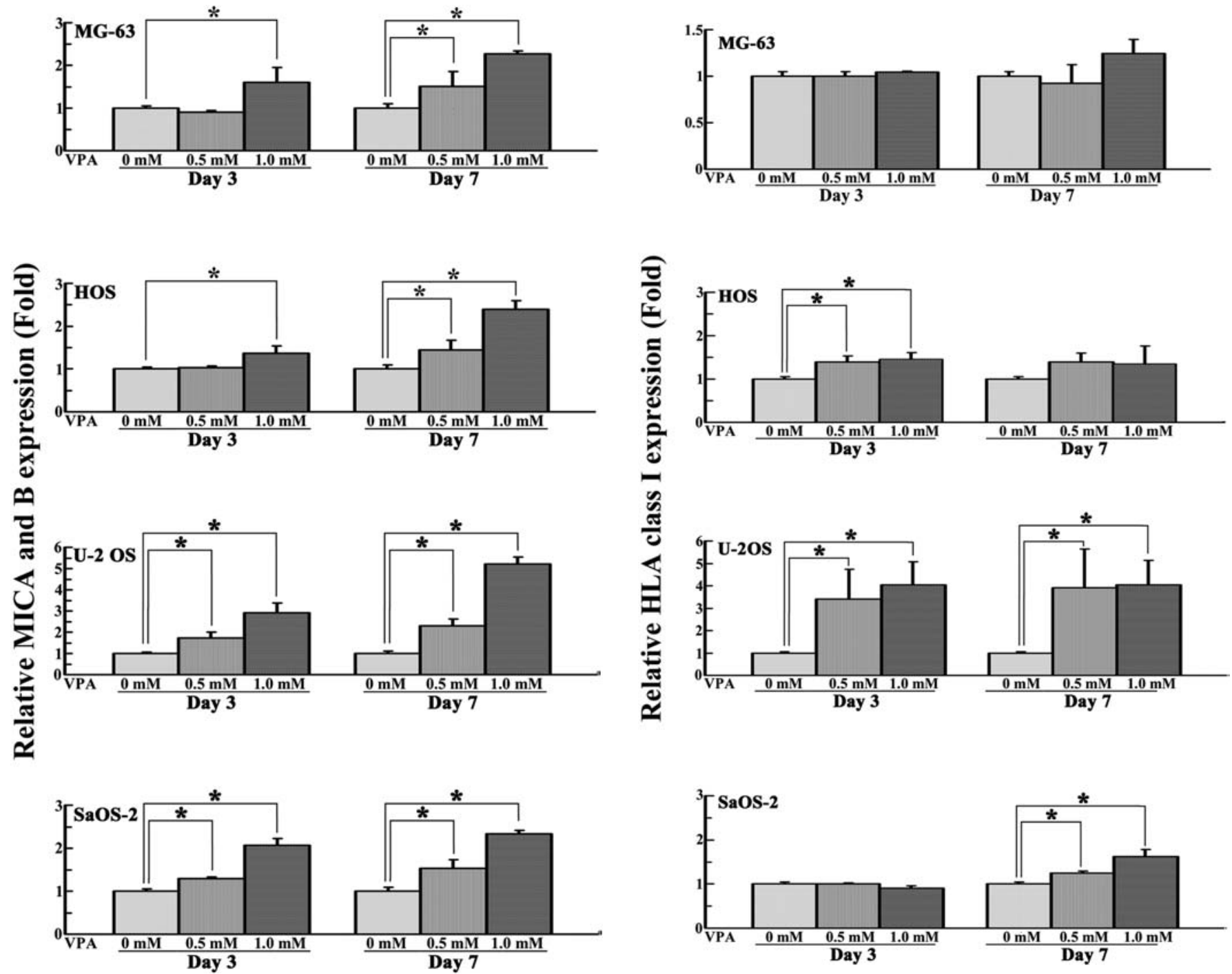

Figure 2. Effects of VPA on the expression of cell-surface MICA and B From flow cytometric profiles of osteosarcoma cells cultured for 3 or 7 days in the presence or absence of 0.5 or $1.0 \mathrm{mM}$ VPA, the effect of VPA on percentage of positive cells was analyzed. The average percentage of positive cells in culture without VPA is expressed as 1. Each bar indicates a mean \pm SE of values obtained from 4 experiments. ${ }^{*} \mathrm{P}<0.05$, significant difference.


Figure 4. Effects of VPA on acetylation of histones bound to MICA and B gene promoters. Chromatin immunoprecipitation analysis was performed with U-2 OS and SaOS-2 cells cultured for 7 days in the presence or absence of 0.5 or $1.0 \mathrm{mM}$ VPA, using the anti-acetyl histone antibody. Immunoprecipitated DNA bound to acetylated histones or DNA bound to acetylated and non-acetylated histones (total DNA) was amplified using specific primers to MICA and MICB promoters. Top panels show amplification of MICA or B promoter DNA bound to acetylated histones. 


\section{Soluble MICA}

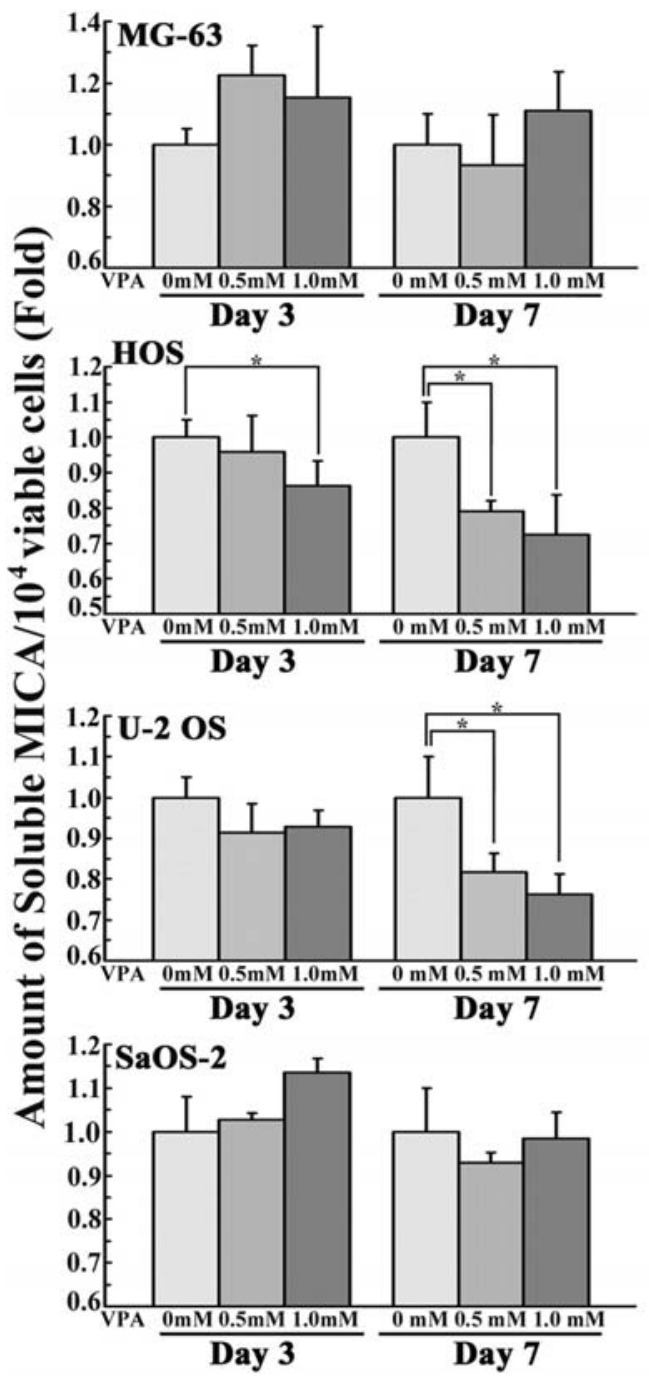

Soluble MICB

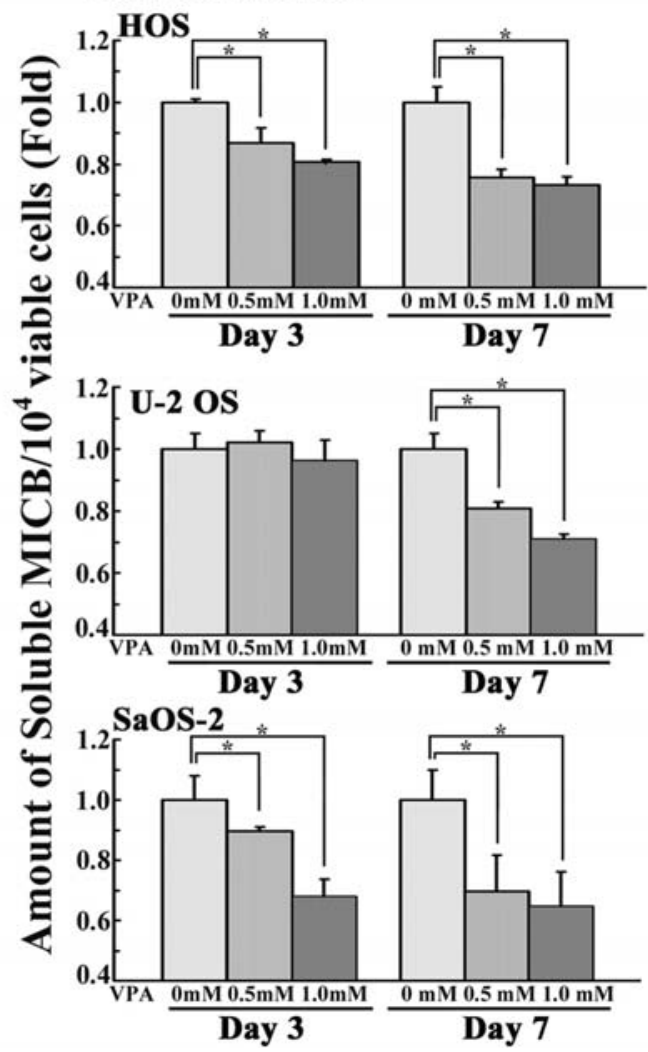

Figure 5. Effects of VPA on the production of soluble MICA and B. Four osteosarcoma cells were cultured in the presence or absence of 0.5 or 1.0 mM VPA for 7 days. The amount of soluble MICA and B accumulated in medium for the first 3 days and the next 4 days was assayed. Their amount per $10^{4}$ viable cells was presented by expressing the average amount per $10^{4}$ viable cells in culture in the absence of VPA as 1 . Each bar indicates the mean \pm SE of 8 dishes. ${ }^{*} \mathrm{P}<0.05$, significant difference.

and $\mathrm{B}$, all four osteosarcoma cells were cultured in the presence of VPA at 0.5 or $1.0 \mathrm{mM}$ for 7 days, and soluble MICA and B accumulated in medium during the first 3 days and the next 4 days were assayed. We found that soluble MICA levels were essentially unchanged in MG-63 and SaOS-2 cells on days 3 and 7 , but markedly decreased in HOS and U-2 OS cells on day 7 in the presence of 0.5 or $1.0 \mathrm{mM}$ VPA (Fig. 5). No soluble MICB was detected in MG-63 cells with or without VPA on days 3 and 7. Soluble MICB levels were decreased on days 3 and 7 in HOS and SaOS-2 cells, and on day 7 in U-2 OS cells in the presence of 0.5 or $1.0 \mathrm{mM} \mathrm{VPA} \mathrm{(Fig.} \mathrm{5).}$

Effects of VPA on the susceptibility to NK cell-mediated cell death. HOS, U-2 OS and SaOS-2 cells were cultured for 7 days in the presence of 0.5 and $1.0 \mathrm{mM}$ VPA and susceptibility to cytotoxicity of NK-92 NK cells was examined. Treatment with VPA at 0.5 and $1.0 \mathrm{mM}$ was found to increase the susceptibility of these three osteosarcoma cells to NK-92 cells (Fig. 6). Pretreatment of NK-92 cells with NKG2Dblocking antibody completely prevented the cytotoxic activity of NK-92 cells against all three target cells treated with $1.0 \mathrm{mM}$ VPA (Fig. 6).

\section{Discussion}

In this study, we showed that VPA elevated the expression of MICA and B on the surface of osteosarcoma cells and enhanced their susceptibility to the cytotoxicity of NK cells as reported with other tumor cells (6-11). On the other hand, levels of soluble MICA and B were not changed or rather decreased in the presence of VPA. In human hepatoma cells, VPA has been reported to increase the production of soluble MICA and B (7). Soluble MICA and B are produced by cleavage of cell-surface MICA and B by proteases including matrix metalloproteinases $(1,2)$. Another report has revealed that this cleavage depends on conformational changes of MIC catalyzed by Erp5 disulfide-isomerase (17). We have 

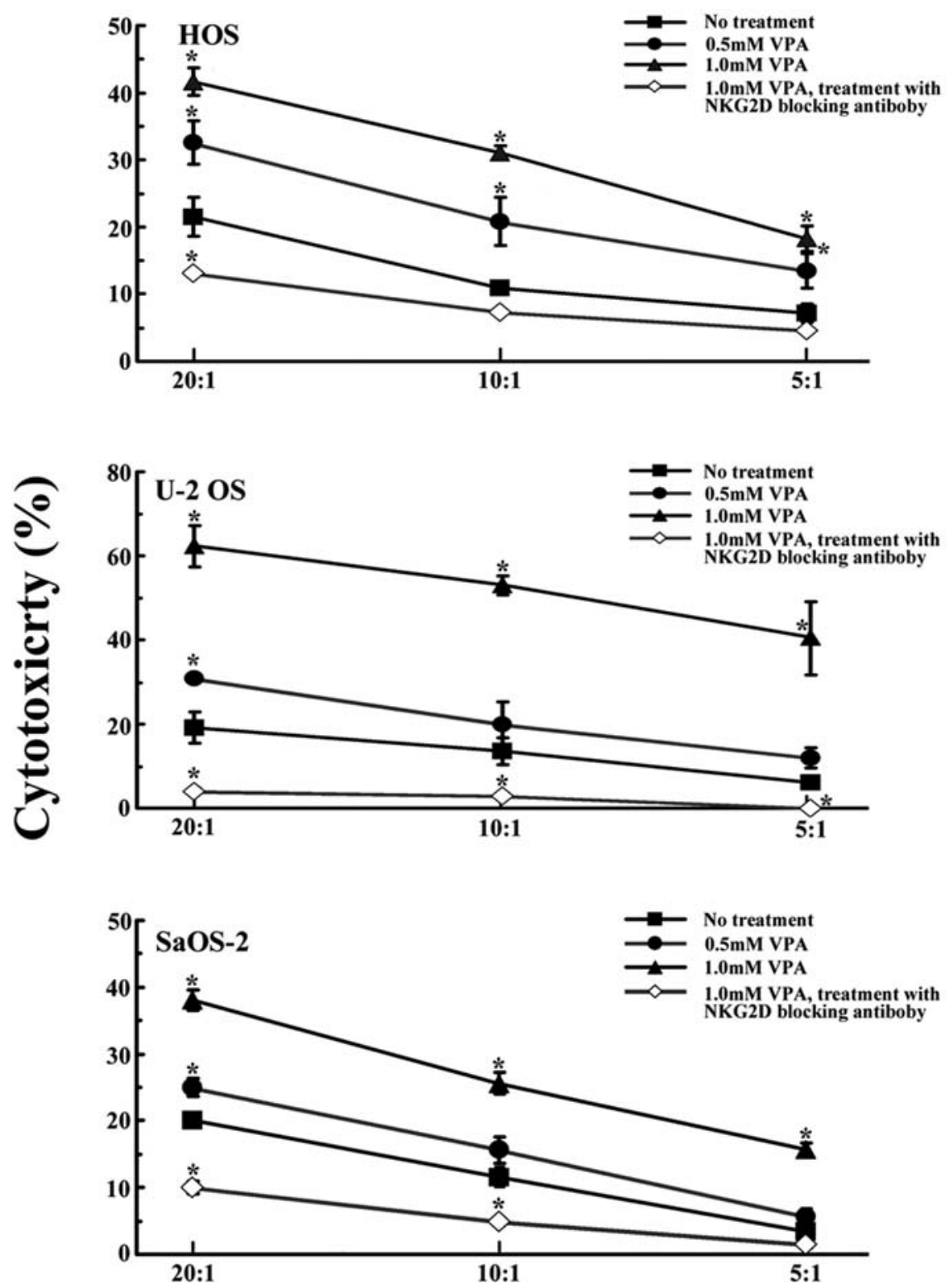

Figure 6. Effects of VPA on the susceptibility of osteosarcoma cells to NK cell-mediated cytotoxicity. Cytotoxic assays were carried out by incubating 3 osteosarcoma cell lines (target cells; T) cultured with or without 0.5 or $1.0 \mathrm{mM}$ VPA for 7 days and NK-92 cells (effector cells; E) at various E/T ratios. In blocking experiments, osteosarcoma cells pretreated with $1.0 \mathrm{mM}$ VPA was incubated with NK-92 cells pretreated with anti-NKG2D antibody for $16 \mathrm{~h}$. Each bar indicates a mean \pm SE of 8 samples. ${ }^{*} \mathrm{P}<0.05$, significant difference from the values of cells cultured without VPA.

investigated whether matrix metalloproteinases participated in production of soluble forms. Our preliminary data showed that VPA decreased mRNAs of some matrix metalloproteinases in osteosarcoma cells (data not shown). Although further studies are needed, it is possible that VPA has effects on the expression of matrix metalloproteinases in osteosarcoma and hepatoma cells.

We reported previously that VPA at 0.5 and $1.0 \mathrm{mM}$ increased the amount of acetylated histones in MG-63, HOS, U-2 OS and SaOS-2 osteosarcoma cells (13). In this study, the chromatin immunoprecipitation assay revealed that VPA enhanced the acetylation of histones bound to MICA and B promoters. Since acetylation status of histones is closely related to gene regulation $(4,5)$, it is possible that the modification of DNA-bound histones described above caused increased expression of cell-surface MICA and B in osteosarcoma cells.
HLA class I molecules suppress the activity of NK cells through inhibitory receptors $(1,18)$. In this study, VPA increased the expression of HLA class I in osteosarcoma cells, particularly in U-2 OS cells. However, VPA also increased the susceptibility of U-2 OS cells to the cytotoxicity of NK cells. These results suggest that the effect of VPA on the amount of cell-surface MICA and B is greater than that on the amount of HLA class I in osteosarcoma cells.

Pretreatment of NK cells with the NKG2D-blocking antibody reduced the cytotoxic activity of NK cells on osteosarcoma cells pretreated with $1.0 \mathrm{mM}$ VPA to levels less than that on osteosarcoma cells without pretreatment. These results suggest that the VPA-induced increase in the expression of NKG2D ligands is involved in increased susceptibility of osteosarcoma cells to NK cells. Human NKG2D ligands consist of not only MICA and B but also 
the UL 16-binding proteins (ULBPs) $(1,2)$. Effect of VPA on ULBP levels was not analyzed in this study. Therefore, we can not exclude the possibilitiy of the contribution of VPAinduced ULBPs to a VPA-induced increase in susceptibility of osteosarcoma cells to NK cells.

NKG2D is expressed on NK cells and other cytotoxic immune cells, such as CD8 ${ }^{+} \mathrm{T}$ cells and $\gamma \delta^{+} \mathrm{T}$ cells $(1,2)$. Therefore, VPA likely enhances the susceptibility of osteosarcoma cells to these cytotoxic immune cells. VPA has also been shown to enhance the susceptibility of osteosarcoma cells to Fas-ligand-induced cell death (13). These results suggest that VPA in combination with immunotherapy activating cytotoxic immune cells could be useful to treat osteosarcomas.

\section{Acknowledgements}

This study was in part supported by a Grant-in-Aid for Young Scientists (B) (21791835) from the Ministry of Education, Culture, Sports, Science, and Technology of Japan, Grant-in-Aid for Researchers, Hyogo College of Medicine, 2008, a Hitec Research Center grant and Grantin-Aid for Promotion of Technical Seeds in Advanced Medicine, Hyogo College of Medicine.

\section{References}

1. Waldhauer I and Steinle A: NK cells and cancer immunosurveillance. Oncogene 27: 5932-5943, 2008.

2. Nausch $\mathrm{N}$ and Cerwenka A: NKG2D ligands in tumor immunity. Oncogene 27: 5944-5958, 2008.

3. Jinushi M, Takehara T, Tatsumi T, Tatsumi T, Hiramatsu N, Sakamori R, Yamaguchi S and Hayashi N: Impairment of natural killer cell and dendritic cell functions by the soluble form of MHC class I-related chain A in advanced human hepatocellular carcinomas. J Hepatol 43: 1013-1020, 2005.

4. Lane AA and Chabner BA: Histone deacetylase inhibitors in cancer therapy. J Clin Oncol 27: 5459-5468, 2009.

5. Glozak MA and Seto E: Histone deacetylases and cancer. Oncogene 26: 5420-5432, 2007.

6. Skov S, Pedersen TM, Andersen L, Straten PT, Woetmann A and Ødum N: Cancer cells become susceptible to natural killer cell killing after exposure to histone deacetylase inhibitors due to glycogen synthase kinase-3-dependent expression of $\mathrm{MHC}$ class I-related chain A and B. Cancer Res 65: 11136-11145, 2005.
7. Armeanu S, Bitzer M, Lauer UM, et al: Natural killer cellmediated lysis of hepatoma cells via specific induction of NKG2D ligands by the histone deacetylase inhibitor sodium valproate. Cancer Res 65: 6321-6329, 2005.

8. Diermayr S, Himmelreich H, Durovic B, et al: NKG2D ligand expression in AML increase in response to HDAC inhibitor valproic acid and contributes to allorecognition by $\mathrm{NK}$ cell lines with single KIR-HLA-class I specificities. Blood 111: 1428-1436, 2008.

9. Schmudde M, Braun A, Pende D, et al: Histone deacetylase inhibitors sensitize tumour cells for cytotoxic effects of natural killer cells. Cancer Lett 272: 110-121, 2008.

10. Poggi A, Catellani S, Garuti A, Pierri I, Gobbi M and Zocchi MR: Effective in vivo induction of NKG2D ligands in acute myeloid leukaemias by all-trans-retinoic acid or sodium valproate. Leukemia 23: 641-648, 2009.

11. Zhang C, Wang Y, Zhou Z, Zhang J and Tian Z: Sodium butyrate upregulates expression of NKG2D ligand MICA/B in HeLa and HepG2 cell lines and increases their susceptibility to NK lysis. Cancer Immunol Immunother 58: 1275-1285, 2009.

12. Imai T, Adachi S, Nishijo K, et al: FR901228 induces tumor regression associated with induction of Fas ligand and activation of Fas signaling in human osteosarcoma cells. Oncogene 22: 9231-9242, 2003.

13. Yamanegi K, Yamane J, Hata M, et al: Sodium valproate, a histone deacetylase inhibitor, decreases the secretion of soluble Fas by human osteosarcoma cells and increases their sensitivity to Fas-mediated cell death. J Cancer Res Clin Oncol 135: 879-889, 2009.

14. Watanabe K, Okamoto K and Yonehara S: Sensitization of osteosarcoma cells to death receptor-mediated apoptosis by HDAC inhibitors through downregulation of cellular FLIP. Cell Death Differ 12: 10-18, 2005.

15. Johannessen CU and Johannessen SI: Valproate: past, present, and future. CNS Drug Rev 9: 199-216, 2003.

16. Allen MH, Hirschfeld RM, Wozniak PJ, Baker JD and Bowden CL: Linear relationship of valproate serum concentration to response and optimal serum levels for acute mania. Am J Psychiatry 163: 272-275, 2006.

17. Kaiser BK, Yim D, Chow IT, et al: Disulphide-isomeraseenabled shedding of tumor-associated NKG2D kigands. Nature 447: 482-486, 2007.

18. Fuertes MB, Girart MV, Molinero LL, et al: Intracellular retention of the NKG2D ligand $\mathrm{MHC}$ class I chain-related gene $\mathrm{A}$ in human melanomas confers immune privilege and prevents NK cell-mediated cytotoxicity. J Immunol 180: 4606-4614, 2008. 